\title{
Newly Decision-making Model of Power Trading Scheme based on Grid Complex Constraints and Power Plants Individual Differences
}

\author{
Jianrong GONG ${ }^{1}$, Guoning GAO ${ }^{1}$, Junli WU ${ }^{2}$, Weijing ZHANG ${ }^{3}$, * $\mathrm{Si} \mathrm{CHEN}^{3}$ \\ ${ }^{1}$ State Grid Zhejiang Electric Power Trade Center, Zhejiang Hangzhou, 310007; \\ ${ }^{2}$ State Grid Zhejiang Economic Research Institute, Zhejiang Hangzhou, 310007; \\ ${ }^{3}$ Beijing Tsingsoft Technology Co., Ltd., Beijing, 100085, China
}

\begin{abstract}
Keywords: power purchase planning; optimization decision-making model; power network constraints; referential progress
\end{abstract}

\begin{abstract}
Power purchase planning is one of the important part of production and operation planning of State Grid. This paper builds an optimization decision-making model of power purchase planning based on grid complex constraints, which can achieve the optimal decision-making of annually and monthly rolling trade planning, can meticulously take all kinds of constraints of power system operation into consideration and consider the relationship between generation schedule and external conditions, such as power network restrictions and deliverability of primary energy. Meanwhile, four types of referential progress are introduced. These four progress adequately consider the unit maintenance, load demand and the preference of progress equilibrium of decision-makers. Then, the newly decision-making model of trade planning based on grid complex constraints and referential progress is built.
\end{abstract}

\section{Introduction}

China started market-oriented reform of electric power industry since year 1998; In year 2002, the State Council issued the [2002] No.5 document 《Notice about publishing scheme of electric system reform》, which explicitly proposed scheme of electric system reform, implementation of "plant and network separation" and "electricity bidding", as well as building of regional electricity market; In year 2015, No.9 document of electric power reform, along with 6 supportive documents were issued, which further promoted the market-oriented process of "distribution and selling separation". With the development of electric power industry, the government has not adapted the demand of electricity market reform, which mainly reflects in restricting the optimal resources allocation of market, inability of forming accurate price signal to embody the electric power supply and demand, as well as inability in leading the optimal power supply layout and user resource investment.

Trade planning establishment of State Grid includes contract electric energy decomposition, as well as power generation and purchase planning establishment. Currently, all contract electric energy of power plants should be decomposed, from year to month and from month to day. Documents [6]-[10] introduce different methods for decomposing contract electric energy, and these methods have certain scenario suitability. Establishment of power generation and purchase planning generally considers purchasing cost, security of power network operation, energy conservation and environment protection, along with power loss as optimization objectives. According to the establishment period, trade planning can be divided into short-term, medium-term and long-term ones. Documents [11]-[13] introduce three kinds of daily generation planning model. Documents [14] and [15] introduce two kinds of monthly generation planning model. Document [16] raises a flexible and expandable method for generation planning establishment with grouping multiple objectives. This method can flexibly allocates optimization objective based on coal consumption, pollution discharge, power loss and purchasing cost. Document [17] obtains the blend curve of power network security and economic operation by optimization of dual objectives, which realizes quantized estimation of the blend cost between economy and safety of power network. 
This paper emphasizes on the risk factors of short-term and medium-term generation planning establishment, including locating the key factors of planning establishment and execution risks; raising the concept of referential progress and introducing optimal decision technology for multi-objectives to rearrange the establishment procedure of power purchasing planning., which provides rich and pointed information for State Grid Zhejiang Electric Power Trade Center to implement and establish trading planning.

\section{Drawbacks of traditional purchasing establishment mode}

State Grid Zhejiang Electric Power Trade Center has already carried out some informative work regarding the power purchasing planning establishment, which to some extent improves the working efficiency and management level. However, as the gradual expansion of business requirement, the current working mode cannot adapt to the new situation, the concrete analysis is as follow:

(1) Planning and decision depend on experience, inadequate consideration of actual constraints

In the process of establishing power purchasing planning, decision-makers always adopt experienced methods, which inadequately considers the unit maintenance, unit output restrictions and primary energy supplement. The irrationalities accumulated till end of the year, leading to big implementation deviation of generation planning of power plants.

(2) Risk management and control mechanism is not introduced

The present establishment mode of power purchasing planning always ignores the uncertainties involved in the production and operation of power system, also not taking the effects of uncertainties and execution errors into consideration. Therefore, the trade planning is always static, which cannot provide comprehensive and accurate referential information for dispatching decision, and cannot perform objective estimation of the effects and risks brought by uncertainties.

(3) Inability in realizing time coordination of power generation planning

Currently, when establishing the monthly generation planning, State Grid Zhejiang Electric Power Trade Center concerns the load rate as the only indicator for estimating the rationality of the subsequent months. Therefore, coordination among months is lack. The execution deviations accumulate month by month, which finally results in big deviations for generation planning at the end of the year.

\section{Decision-making model for power purchasing planning considering complex constraints of power network}

Based on the above analysis, this paper builds the decision-making model for power purchasing planning by considering the complex constraints of power network. This model regards the monthly generation planning as the decisive parameter, which can realize optimal decision-making of annually and monthly rolling trade plan, can achieve coordinated optimization and layered optimization of multiple objectives, can meticulously take all kinds of constraints of power system operation into consideration and consider the relationship between generation schedule and external conditions, such as power network restrictions and deliverability of primary energy.

\subsection{Decisive parameters}

The decisive parameters of power purchase planning are the monthly generation planning of each power plant and the monthly external purchase planning. In this paper, $Q_{P_{i}}^{m}$ stands for monthly generation planning of each power plant and $Q_{X_{j}}^{m}$ stands for monthly external purchase planning.

\subsection{Optimization objective}

\subsubsection{Economic objective}

$$
\min \sum_{m=T_{m}}^{T_{M}}\left[\sum_{i=1}^{N_{P}} c_{P_{i}}^{m}\left(1-\alpha_{P_{i}}^{m}\right) Q_{P_{i}}^{m}+\sum_{j=1}^{N_{X}} c_{X_{j}}^{m}\left(1-\beta_{X_{j}}^{m}\right) Q_{X_{j}}^{m}\right]
$$


In equation (2-1), $T_{m}$ is the starting month; $T_{M}$ is the ending month; default value $T_{M}=12 ; N_{P}$ is the number of power plants; $c_{P_{i}}^{m}$ is the average feed-in tariff of power plant $i$ in month m; $\alpha_{P_{i}}^{m}$ is the average power consumption rate of power plant $i$ in month $\mathrm{m} ; \beta_{X_{j}}^{m}$ is the loss factor of tie lines.

The objective function aims at the total cost of trade planning is minimum, the first part stands for the total cost for purchasing the on-grid power; the second part stands for the total cost for purchasing the external power.

\subsubsection{Energy-conserved objective}

$$
\min \sum_{m=T_{m}}^{T_{M}} \sum_{i=1}^{N_{P}} h_{P_{i}}^{m} Q_{P_{i}}^{m}
$$

In equation (2-2), $h_{P_{i}}^{m}$ is the average coal consumption of power plant $i$ in month $m$. The objective function aims at the total coal consumption of all the power plants is minimum.

\subsubsection{Balanced objective}

$$
\min \sum_{m=T_{m}}^{T_{M}} \sum_{i=1}^{N_{P}}\left|\rho_{P_{i}}^{m}-\frac{1}{N_{P}} \sum_{i=1}^{N_{P}} \rho_{P_{i}}^{m}\right|
$$

\subsubsection{Objective of completion rate for year planning}

$$
\min \sum_{i=1}^{N_{P}}\left|\rho_{P_{i}}^{Y}-\frac{1}{N_{P}} \sum_{i=1}^{N_{P}} \rho_{P_{i}}^{Y}\right|
$$

In equation (2-3), $\rho_{P_{i}}^{m}$ stands for the accumulated generation progress for power plant $i$ in month $m, \rho_{P_{i}}^{m}$ represents as:

$$
\rho_{P_{i}}^{m}=\frac{\sum_{m^{\prime}=1}^{m} Q_{P_{i}}^{m^{\prime}}}{Q_{P_{i}}^{Y}}
$$

For months that happened, $Q_{P_{i}}^{m}$ is the actual electric power generation for each month, which is the known quantity; for months that not happened, $Q_{P_{i}}^{m}$ is decisive quantity; $Q_{P_{i}}^{Y}$ is the generation planning of power plant $i$; $\rho_{P_{i}}^{Y}$ is the completion rate of yearly generation planning of power plant, and obviously it equals to the accumulated generation rate at December, which can be represented as:

$$
\rho_{P_{i}}^{Y}=\left.\rho_{P_{i}}^{m}\right|_{m=12}
$$

Balanced objective and objective of completion rate for year planning aim at the fairness and balance of generation planning of each power plant. The difference is that balanced objective purposes at the balance and synchronization of generation planning for each power plant during the whole year, which belongs to process equilibrium; objective of completion rate for year planning purposes at completion of yearly generation planning consistently at the end of the year for each power plant This objective belongs to target equilibrium and in actual production, deviation no more than $2 \%$ is preferred.

Generally, to trade center in State Grid, economic and energy-conserved objectives are basically determined by the year generation planning, and the space for optimization is very limited. Balanced objective and objective of completion rate for year planning are the key ones that trade center concerns mostly, in these two, the objective of completion rate for year planning is the primary objective. Meanwhile, the progress equilibrium should be controlled during the whole procedure.

\subsection{Constraints}

\subsubsection{Power system equilibrium constraint}

Power balance should be ensured, and in trade planning is mainly represented as electric power balance. Based on the comprehensive consideration of network loss and power plant consumption, sum of electric energy from unified management power plants, local power plants and tie lines 
should equal to the electric power sales.

$$
\left\{\begin{array}{c}
\sum_{i=1}^{N_{P}}\left(1-\alpha_{P_{i}}^{m}\right) Q_{P_{i}}^{m}+\sum_{j=1}^{N_{X}}\left(1-\beta_{X_{j}}^{m}\right) Q_{X_{j}}^{m}=Q_{D}^{m}+Q_{L}^{m} \\
\forall m
\end{array}\right.
$$

In equation (2-7), $Q_{D}^{m}$ is electric power sales; $Q_{L}^{m}$ is electric power loss.

\subsubsection{Power network constraint}

The security for key elements and sections should be properly constrained.

$$
\left\{\begin{array}{c}
\sum_{i \in \Omega_{k}} d_{P_{i}, \Omega_{k}} Q_{P_{i}}^{m} \leq \bar{Q}_{\Omega_{k}}^{m} \\
\forall k
\end{array}\right.
$$

In equation (2-8), $\Omega_{k}$ is a set for security constraints; $d_{P_{i}, \Omega_{k}}$ is the distribution factor of shifting electric energy; $\overline{Q_{\Omega_{k}}}$ is the upper limit of electric power under security constraint. It should be noted that this constraint should be more precisely expressed in electric format, however, there are several difficulties in actual decisions, and therefore, the electric energy is used as an approximation.

\subsubsection{Stock coal constraint}

The monthly coal consumption of power plants should be less than the stock coal, in general, satisfaction of generation demand by quantity of stock coal is considered.

$$
\left\{\begin{array}{l}
Q_{P_{i}}^{m} h_{P_{i}}^{m} \leq S_{P_{i}} \\
m=T_{m}, \forall i
\end{array}\right.
$$

In equation (2-9), $S_{P_{i}}$ is the stock coal.

\subsubsection{Electric energy constraint}

According to the unit capacity and maintenance schedule, the adjustable output and maximum generation capability for each power plant at each month can be determined.

$$
\begin{aligned}
& C_{P_{i}}^{m}=\sum_{j=1}^{G_{P_{i}}}\left(1-\frac{D_{P_{i}, G_{j}}^{m}}{T_{D}^{m}}\right) C_{P_{i}, G_{j}} \\
& M_{P_{i}}^{m}=C_{P_{i}}^{m} T_{D}^{m} T_{H}
\end{aligned}
$$

In equations (2-10) and (2-11), $C_{P_{i}}^{m}$ is the adjustable output for power plant $i$ in month $m$; $M_{P_{i}}^{m}$ is the maximum generation capability for power plant $i$ in month $m$; $G_{P_{i}}$ is the number of unit for power plant $i$; $D_{P_{i}, G_{j}}^{m}$ is the days for maintenance of unit $\mathrm{j}$ in power plant $i$ in month $m$; $T_{D}^{m}$ is the natural days in month $m ; C_{P_{i}, G_{j}}^{m}$ is the capacity for unit $\mathrm{j}$ in power plant $i$; $T_{H}=24 h$, which is the number of hours in a day.

In actual production, because of some factors, such as system backup, heat supply and capacity restriction, the variation range for power plants should be specified, which is usually achieved by specifying the range of unit energy or load rate. This kind of constraint is universality and global, as well as personalized, which is expressed as follows:

$$
\left\{\begin{array}{c}
\underline{Q}_{P_{i}}^{m} \leq Q_{P_{i}}^{m} \leq \bar{Q}_{P_{i}}^{m} \\
\forall m, i
\end{array}\right.
$$

In equation (2-12), $\underline{Q}_{P_{i}}^{m}$ and $\bar{Q}_{P_{i}}^{m}$ are the upper and lower generation limits by considering the restrictions of power plant operation.

\subsubsection{Power generation planning constraint}

During the process of establishment of power generation planning, the ability of power plants to complete the yearly generation planning of the subsequent months should be carefully considered. 
The electric energy should be properly arranged, which ensures all the power plants can complete the yearly generation planning equally. However, when the establishment of yearly generation planning is unreasonable, the adjustment of yearly contract energy should be allowed (generally, the permissible deviation is $2 \%$ ).

$$
\left|\rho_{P_{i}}^{Y}-100 \%\right| \leq 2 \%
$$

In general, this constraint can be satisfied and it is a flexible constraint.

\section{The decision-making model of power purchase planning based on referential progress}

In Section 2.2.2, a balanced optimization decision-making model for power purchase planning was put forward in Equation (2-3). This model treats the progress deviation of all power plants indistinguishably, which may cause large progress variance for big power plants, as a result, providing optimization space for small power plants. In addition, the model needs to calculate the average progress of all the power plants. For large-scale planning, this modeling method greatly increases the amount of calculation, which was not conducive for solving problems. And, take the average progress of all power plants as the calculation benchmark of the progress deviation, which ignores the individual differences between power plants, such as monthly maintenance situation of power plants and heating demand.

To summarize the above shortcomings, this paper puts forward the concept of referential progress.

\subsection{The concept of referential progress}

For the balanced objective function of power purchase planning, choice of reasonable benchmark, namely referential progress to compute the progress deviation is the important premise to guarantee the rationality and scientific nature of the power purchase planning. Reasonable referential progress should at least have the following characteristics:

(1) Referential progress of all power plants is the same at the end of the year. Under the condition of annual generation planning and separable year planning are in balance, the referential progress of all power plants clearly should be $100 \%$ at the end of the year;

(2) Full reflection of differences in capacity for different power plants at different months, which embodies the overhaul of power plants. For months that have overhaul happened, referential progress should slow down correspondingly;

(3) Full reflection of changes of grid load level, which embodies the changes of separable month planning. For months with large load, generation of power plants should also increase accordingly, so the referential progress will speed up correspondingly.

\subsection{The four setting methods for referential progress}

In this paper, four setting methods of referential progress are raised, and preliminarily analyze and compare the advantages and disadvantages of each method. It should be pointed out that, the four setting methods are extracted from different engineering practice, and each has its own advantages and disadvantages. With considering the influences of many factors and the difference of trading scheme decision, each setting method of the referential progress can only be "relatively reasonable", and cannot meet the requirements of "absolutely reasonable".

(1)Average progress of the whole network(GAP)

Define the average progress of the whole network as:

$$
\mathrm{GAP}=\frac{\sum_{m^{\prime}=1}^{m} Q_{G}^{m^{\prime}}}{Q_{G}^{Y}}
$$

In equation (3-1), $Q_{G}^{m}$ is the total power generation planning in month $m$ (decomposable planning); $Q_{G}^{Y}$ is the annual total power generation planning (decomposable planning).

(2)Preferential progress for power plants(PPP)

Define preferential progress of power plant $i$ in month $m$ as: 


$$
\mathrm{PPP}=\frac{\sum_{m^{\prime}=1}^{m} M_{P_{i}}^{m^{\prime}}}{M_{P_{i}}^{Y}}
$$

In equation (3-2), $M_{P_{i}}^{m^{\prime}}$ is the maximum power generation capacity of power plant $i$ in month $m$, which is the generation capacity under the full generation of the adjustable capacity during the whole month; $M_{P_{i}}^{Y}$ is the maximum annual generating capacity of power plant $i$, which is sum of the monthly maximum generating capacity.

(3)Average progress of the whole network considering adjustable capacity of power plants(CMGAP)

According to the deficiency of the average progress of the whole network, it is revised through the adjustable capacity of power plants. The average progress of the whole network based on the adjustable capacity of power plants is defined as:

$$
\text { CMGAP }=\frac{\sum_{m^{\prime}=1}^{m} Q_{G}^{m^{\prime}}}{Q_{G}^{Y}} \cdot \frac{\sum_{m^{\prime}=1}^{m} C_{P_{i}}^{m^{\prime}}}{m \times \bar{C}_{P_{i}}}
$$

In equation (3-3), $C_{P_{i}}^{M}$ is the adjustable capacity of power plant $i$ in month $m$; $\overline{C_{P_{i}}}$ is the average adjustable capacity of the power plant $i$. The second right part of equation (3-3) is called the correction coefficient of adjustable capacity.

(4)Preferential progress for power plants considering the modification of the whole network load(LMPPP)

Aiming at the shortcomings of the preferential progress of power plants, using the whole network load level to modify the preferential progress and is defined as:

$$
\text { LMPPP }=\frac{\sum_{m^{\prime}=1}^{m} M_{P_{i}}^{m^{\prime}}}{M_{P_{i}}^{Y}} \cdot \frac{\sum_{m^{\prime}=1}^{m} q_{G}^{m^{\prime}}}{m \times \bar{q}_{G}}
$$

In equation (3-4), $q_{G}^{m}$ is the load level of the whole network in month $m$, which can be obtained by division of separable plan of month $m$ by number of days; $\overline{q_{G}}$ is the average load level of the whole network. In this paper, the second right part of equation (3-4) is called correction coefficient of load level.

Table 3.1 summarizes advantages and disadvantages of the four referential progress.

Table 3.1 Comparison of Four Referential Progress

\begin{tabular}{|c|c|c|}
\hline Referential Progress & Advantages & Disadvantages \\
\hline GAP & $\begin{array}{c}\text { Network load demand have the priority, } \\
\text { absolutely fair }\end{array}$ & $\begin{array}{c}\text { Ignore the overhaul of power } \\
\text { plant }\end{array}$ \\
\hline PPP & $\begin{array}{c}\text { Power plant capacity have the priority, } \\
\text { absolutely fair }\end{array}$ & Ignore the load demand \\
\hline CMGAP & $\begin{array}{c}\text { Overall consideration of load demand } \\
\text { and power plant capacity }\end{array}$ & $\begin{array}{c}\text { Impact by change of } \\
\text { maintenance planning }\end{array}$ \\
\hline LMPPP & $\begin{array}{c}\text { Overall consideration of load demand } \\
\text { and power plant capacity }\end{array}$ & $\begin{array}{c}\text { Impact by accuracy of load } \\
\text { forecasting }\end{array}$ \\
\hline
\end{tabular}

\subsection{Decision-making Model}

Based on Section 2.2 and Section 3.1, this paper proposes a newly decision-making model for power trade scheme based on referential progress of power plants. The unified optimal decision-making model can be expressed as:

$$
\min \sum_{m=T_{m}}^{T_{M}} \sum_{i=1}^{N_{P}} W_{P_{i}}^{m}\left|\rho_{P_{i}}^{m}-\bar{\rho}_{P_{i}}^{m}\right|
$$


In Equation (3-5), $W_{P_{i}}^{m}$ is the weight of progress deviation for power plant $i$ in month $m$; $\bar{\rho}_{P_{i}}^{m}$ is the referential progress for power plant $i$ in month $m$.

The model takes into account the overhaul situation of each power plant in different months, the load demand of the whole network and the balanced progress preference. Meanwhile, the model is compatible with the decision model of power purchase planning in section 2.2 which considers complex constraints of power network.

\section{Conclusion}

Aimed at the current problems and shortcomings of trading scheme for State Grid Zhejiang Electric Power Trade Center. Firstly, a transaction planning decision-making model considering the complex constraints of the power grid is constructed. The model realizes the optimal decision for the trading planning of the whole year and multi-month rolling and multi-objective coordination optimization, as well as the layered optimization. On this basis, the concept of referential progress is proposed, and a newly decision-making model for trade scheme based on referential progress is constructed. The model effectively improves the balance of decision-making, coordinates differences between power plants, as well as among months, which finally provides enough margin space for dispatching, and avoids the risk of extreme scenarios.

\section{References}

[1] DU Songhuai. Power Market (Third Edition) [M].Beijing: China electric power press, 2008.

[2] ZHANG Caiqing, HUANG Ying. Risk Assessment for Power Grid Electricity Purchasing Based on Venture Profit Constraint [J]. Central China Electric Power, 2010(3):68-71.

[3] SHI Quansheng. Risk Analysis of Purchasing Electricity to Grid Company Based on Assert Combination Theory [J].Power System Technology, 2008(S1):137-138.

[4] Dahlgren R, Chen-Ching L, Lawarree J. Risk assessment in energy trading [J]. IEEE Transactions on Power Systems, 2003, 18(2):503-511.

[5] Mitani T, Mishima Y, Satoh T, et al. Optimal Generation Scheduling under Competitive Environment: IEEE International Conference on Systems, Man, and Cybernetics, Taipei, Taiwan, 2006[C].

[6] DAI Tiechao, ZHANG Dan. Application of Resoluting Computation of Decisive Contract Electric Energy in Zhejiang Generation Power Market [J].East China Electric Power, 2000(10):7-9.

[7] CHEN Jianhua, ZHANG Ning et al. Empirical Analysis on the Deterministic Contract Decomposition Algorithm [J]. Automation of Electric Power Systems, 2008, 32(2):21-25.

[8] LI Canbing, HU Yajie, ZHAO Hongjun et al. General Model and Algorithm for Contract Energy Decomposition [J]. Automation of Electric Power Systems, 2007, 31(11):26-30.

[9] WANG Shuai. Research on Optimization Algorithm of Generation Rolling Plan [D]. Beijing: North China Electric Power University (Beijing), 2009.

[10]SU Pengfei. Research on the Decomposition Strategy of Contract Quantity Considering Uncertainty [D].Shan dong: Shan Dong University, 2010.

[11] SHEN Yu, XIA Qing et al. A Novel Model of Short-term Trade Planning in a Deregulated Power System [J]. Automation of Electric Power Systems, 1999, 23(18):12-16.

[12] WANG Yanling, ZHANG Lizi et al. Coordination of the Dispatching Relationship Between the Contract Volumn and Competitive Bidding Volumn in Daliy Generation Scheduling[J]. Automation of Electric Power Systems, 2002(9):45-48.

[13] LIANG Zhifei, XIA Qing. An Accurate Daily Generation Scheduling Model and Application 
Algorithm [J]. Automation of Electric Power Systems, 2008, 32(17):26-29.

[14] MAO Yi, CHE Wenyan. Monthly Generation Scheduling Considering Energy-saving and Economic Benefit [J]. Modern Electric Power, 2008, 25(5):73-78.

[15] LIANG Zhifei, XIA Qing et al. Monthly Generation Scheduling Method Based on Multi-Objective Optimization Model for Provincial Power Grid [J]. Power System Technology, 2009, 33(13):90-95.

[16]WANG Liangyaun, LIU Wenbin et al. Method and Application of Medium and Long Term Electric Power Purchase Plan Based on Multi Object Grouping [J]. Power System Technology, 2011, 35(6):167-170.

[17] SUN Xin, LÜ Yuechun et al. Power Grid Economy and Security Coordination Lean Method [J]. Power System Technology, 2009(11):12-17.

[18]WANG Jianhui. Analysis of Power Purchase Risk Model in Electricity Market [J], Power System Technology, 2005(9):46-49. 